\title{
Editorial
}

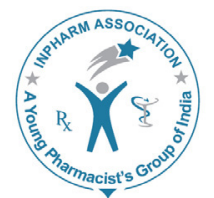

\section{Do antioxidants promote cancer growth?}

The health benefits and therapeutic effects of the antioxidants are well known. The therapeutic effect of the drug is based on its therapeutic risk-and-benefit ratio. "No drug is safe and no poisons are too dangerous", because the safety or dangerous/toxic/undesired effects of any substance based on its dose and route of ingesting. Many of the drugs are used for the homicidal purposes and many of the poisonous substances used for the therapeutic purposes by humankind.

Antioxidants are believed to have high nutritional value and therapeutic effects. Many of the researcher also provided therapeutic efficacy of the antioxidants. Free radical and antioxidant is a balanced system and both having grater physiological effect on the host. Free radical generations are stimulated by carcinogens or by external macromolecules and thereby causing oxidative stress. Inhibition of normal free radical generation process by any of the antioxidant may leads to further inhibition of much of physiological actions which includes redox homeostasis. ${ }^{2}$ In higher organisms, free radicals like nitric oxide, superoxide anion, and relative oxygen species play an important role as regulatory mediators in signaling processes. ${ }^{3}$

As far as the long-term data on adverse effect of antioxidant therapy is not well documented and the maximum of the studies the effect of antioxidant was studied with minimum number of subjects. ${ }^{4}$ Since much of the antioxidant are natural food substances and used at therapeutic or less than therapeutic dose as supplementary therapy along with conventional therapy. Recently few of the authors reported the adverse health effects of antioxidant, which has significant contribution in biomedical research.

Till 2013, many of us believed that antioxidants has higher nutritional value, which may helpful to control/ inhibit the generation of free radicals thereby modulate the progression of cardiovascular systemic and, inflammatory disorders and cancer. But the recent study published by Sayin et al., suggested that antioxidant can accelerate the lung cancer progression rate in rodents, this may be true and same can be happen in human also. ${ }^{5}$ Reduction in relative oxygen species levels may increases the proliferation rates and decreases the expression of the protein (genomic watchdog protein $\mathrm{p} 53$ ), thereby increase the tumor growth. ${ }^{6}$

Lu et al., were reported the antioxidant induced cellular necrosis through a reductive mechanism. Lu et al., were studied the antioxidant effect of Epigallocatechin gallate (EGCG) against human lung diploid fibroblasts (WI-38 and MRC-5 cell lines), skin diploid fibroblast (GM05757 cell line) and Chinese hamster ovary (CHO-K1: GM15452) cell line and found the lethal effects of EGCG. ${ }^{7}$ Free radical destroying supplementary antioxidant may increase the severity of the cancer. In 1994, a 93-year old patient died with prostate cancer patient has taken $12 \mathrm{~g}$ of vitamin $\mathrm{C}$ every day. ${ }^{8}$

The recent studies demonstrates the adverse health effects of antioxidants and further studies are required to confirm the therapeutic and other health effects of the antioxidant. In general, taking antioxidants pills as supplementary by normal individual without consultation of physician is not advisable and not good for a health.

\section{REFERENCES}

1. Tiwari AK. Adverse effects of antioxidants. Current Science, 2003;85:1117.

2. Lecarpentier Y. Physiological role of free radicals in skeletal muscles. J Appl Physiol 2007;103:1917-1918.

3. Parasuraman S, Maithili KS. Antioxidant and drug metabolism. Free Rad Antiox 2014;4:1-2.

4. Chattopadhyay MK. Adverse effects of antioxidants. Current Science, 2003;85:121.

5. Sayin VI, Ibrahim MX, Larsson E, Nilsson JA, Lindahl P, Bergo MO. Antioxidants accelerate lung cancer progression in mice. Sci Transl Med. 2014;6:221ra15.

6. Andrew RM, James WL. Paradoxical Effects of Antioxidants on Cancer. Rejuvenation Res. 2014;17:306-311.

7. Lu LY, Ou N, Lu QB. Antioxidant induces DNA damage, cell death and mutagenicity in human lung and skin normal cells. Sci Rep. 2013;3:3169.

8. Watson J. Oxidants, antioxidants and the current incurability of metastatic cancers. Open Biol. 2013;3:120144.

Dr. Subramani Parasuraman

DOI : 10.5530/jyp.2015.1.1 